\title{
Motivos de no vacunación: un análisis crítico de la literatura internacional, 1950-1990
}

\author{
Motives for non-vaccination: critical review of the international \\ literature, 1950-1990
}

\author{
Gustavo Nigenda-López, Emanuel Orozco y René Leyva \\ Centro de Investigación en Sistemas de Salud. Instituto Nacional de Salud Pública, Morelos-México
}

\begin{abstract}
Resumen
En muchos países se reconoce que los programas de vacunación requieren del dominio de elementos técnicos y organizacionales para ser accesibles a las poblaciones. Uno de ellos ha sido menospreciado de manera importante: la participación de la población y las causas que la motivan. El presente trabajo analiza experiencias de diversos países, a partir de una revisión bibliográfica del período 1950-1990. Los resultados muestran que los estudios existentes varían en su enfoque metodológico y conceptual de acuerdo a la región del mundo en donde fueron realizados y de acuerdo al tipo de investigador. Este hecho se explica por el supuesto que existe en muchos investigadores de pensar que conocen a profundidad los determinantes ideológicos de la conducta de las culturas de las que son nativos. A partir de ello utilizan metodologías que apenas les permiten aproximarse a un nivel superficial del conocimiento de la realidad social para hacer inferencias de la respuesta de las poblaciones ante la oferta de las vacunas.
\end{abstract}

Programas de inmunización, organización. Participación comunitaria.

\begin{abstract}
Many countries have acknowledged that vaccination programs call for a mastery of technical and organizational elements if they are to become accesible to the population. One of these elements has been greatly understimated: the participation of populations and their motivations. Experiences in several countries are here analysed, on the basis of a bibliographic revision of the period 1950-1990. Results show that existing studies vary in their conceptual and methodological focuses, according to the region in which research was carried out and to the kind of researcher involved. This fact is to be explained by the posture, common among researchers, of believing that they know in depth the subjective determinants of the behavior of the societies to which they belong. Based on this, they only use methodologies that allow them to arrive at a superficial understanding regarding the response of populations to the offer of vaccines.
\end{abstract}

Immunization programs, organization. Consumer participation.

Correspondência para/Correspondence to: Gustavo Nigenda-López - Ave. Universidad nº 655, Sta. María Ahuacatitlá, 62508 Cuernavaca, Morelos México. E-mail: gnigenda@insp3.insp.mx

Recebido em 6.10.1995. Reapresentado em 16.8.1996. Aprovado em 20.9.1996. 


\section{INTRODUCCIÓN}

El presente trabajo presenta un análisis sobre una selección de estudios publicados entre 1950 y 1990 que se interesaron por entender la respuesta de las poblaciones ante la oferta de vacunas. Sin duda, el interés por explicar la participación de las poblaciones en los programas de vacunación y los aspectos sociales y culturales que la motivan es escaso y esto se debe en gran medida al éxito que los programas de vacunación han tenido en alcanzar sus metas de cobertura.

Existen datos contundentes sobre el éxito de las campañas de vacunación y su impacto sobre la salud de las poblaciones infantiles en el mundo ${ }^{17}$. En general los programas han desarrollado una gran capacidad para inducir la aceptación de las vacunas por parte de la población. Sin embargo, históricamente se conocen distintos casos en los que las poblaciones han rechazado la oferta de vacunas o condicionado su participación en los programas.

Diversos investigadores se han preguntado sobre cuáles son los elementos sociales y culturales que subyacen a la aceptación de las vacunas por parte de la población a fin de entender hasta dónde esta aceptación tiene un carácter permanente. Desde luego existen una gran cantidad de respuestas que dependen por un lado de los elementos culturales y sociales que caracterizan a las poblaciones, y por otro a la forma en la que los investigadores han abordado el tema.

Como se sabe, ninguna de las vacunas disponibles tiene $100 \%$ de efectividad así como tampoco están exentas de posibles riesgos. Sobre todas ellas existen dudas 'científicas' y dudas 'populares', ya sea por los efectos negativos que puedan producir o porque no produzcan ninguno, ni siquiera protección contra la enfermedad. En el terreno de las vacunaciones "la prudencia es una herramienta esencial", tal como lo afirmó Donald Henderson, coordinador general de la campaña de la erradicación mundial de la viruela en los años 70. Sin embargo, las vacunas suelen despertar en los tomadores de decisiones una sensación de confianza excesiva que sólo puede dar esta arma poderosa cuando no se le identifican puntos de cuestionamiento. Esta sensación de confianza extrema suele hacer que en los círculos tecnocráticos, desde donde se organizan los programas, la opinión de la población receptora de vacunas, no represente un problema a considerar para su buen desarrollo.

En los siguientes apartados se presenta una selección de estudios que se han interesado en la investigación de la respuesta de la población ante los programas de vacunación a fin de evaluar sus resultados para la definición de políticas futuras de vacunación. El lector interesado podrá encontrar un amplio listado de estudios sobe el tema en Heggenhougen y Clements J. ${ }^{22}$. Los estudios fueron agrupados de acuerdo a dos criterios: a) grupo de paises y b) los factores identificados como motivo de no vacunación para el período 1950-1990.

\section{ESTUDIOS EN PAÍSES DESARROLLADOS}

En este grupo de países se incluyen artículos de Estados Unidos, Gran Bretaña e Italia. Los principales factores que se identificaron fueron aquellos relacionados con la política sanitaria, los aspectos psicológicos y el papel del personal de salud como proveedores de información.

\section{Política Inmunizatoria}

En la denominación "política inmunizatoria" se incluyen principalmente aspectos referidos a la organización y las estrategias diseñadas para lograr coberturas en la población. Las campañas de vacunación masiva iniciaron en los Estados Unidos en la primera mitad de la década de los cincuenta con el propósito de "controlar la poliomieltis ${ }^{19}$ ". La estrategia de organización fue de corte militar con el fin de lograr amplia coberturas; sin embargo meses después de haber iniciado la campaña, aún no se obtenía el $85 \%$ de cobertura requerida a nivel nacional para asegurar su éxito. Esta situación se volvió aún más compleja en 1955, cuando cientos de niños desarrollaron parálisis en diversos grados después de haber sido inoculados por un lote de vacuna Salk que no fue preparada con el virus desactivado ${ }^{41}$. Este incidente conocido como "accidente Cutter" es uno de los referentes obligados para comprender el rechazo de la población a las vacunas en esa época. Glasser ${ }^{19}$ mostró que en las regiones con más baja cobertura, la población refería el accidente Cutter como un causa importante de rechazo a la vacunación.

En ese contexto, D'Onofrio ${ }^{14}$ incorporó por primeira vez el concepto de cultura al análisis de las causas de no vacunación. A partir de observaciones realizadas en el sur California, identificó la existencia de grupos de población que definió como "difíciles de alcanzar" para el sistema de salud estatal. Estos grupos se caracterizaban por formar parte de la franja marginal de la sociedad californiana. El estudio no intentó cuantificar motivos de no vacunación, más bien se interesó por detallar la influencia determi- 
nante que las percepciones, los valores y otros elementos de la cultura de dichas poblaciones, tenían sobre su comportamiento ante las vacunas.

En los años 70, la preocupación de Marcuse ${ }^{28}$ se relacionaba con la alta responsabilidad que se exigía a los trabajadores de la salud en norteamérica para generar una mayor demanda de vacunas. Los resultados de algunas encuestas de rutina mostraban la falta de vacunación de "amplios grupos de población".

\section{Cultura y Mitos de la Medicina}

Cabe destacar que en los países industrializados existen comportamientos similares a los documentados en los países en desarrollo en relación a la existencia de mitos e ideas falsas sobre las vacunas. Así, en Italia, la aceptación de la vacuna antisarampionosa hasta al final de los años 80 era particularmente baja. En virtud de este hecho, Profeta y col. ${ }^{38}$ estudiaron este fenómeno a través de entrevistas con madres en la ciudad de Milán. Las razones más frecuentes de falta de vacunación en 1.247 niños fueron las siguientes: ignorancia sobre la vacuna (38\%), creencia de que el sarampión es una enfermedad inocua (36\%). A ellos siguieron en una menor proporción razones como miedo a las reacciones vacunales.

Otro estudio en un pequeño poblado del norte de Italia ${ }^{20}$ mostró que de las personas que no aceptaban la vacuna, $35,7 \%$ declararon estar firmemente opuestos a ella por decisión personal o por consejo médico, 21,4\% consideró la vacuna como innecesaria y $42,9 \%$ la rechazó por enfermedad del niño o por problemas de tiempo. Por su parte, Dianese ${ }^{13}$ señala que la pobre aceptación del componente antitosferínico de la DPT en ese país podría estar asociado a la difundida creencia en amplios sectores de la población de que la tosferina es una enfermedad para la cual no existe tratamiento y que por tanto representa una experiencia obligatoria para los niños.

\section{Información Sobre la Vacunas}

Un patrón importante de comportamiento en países industrializados ha sido el denominado "rechazo informado" que aparece vinculado al manejo de la información por parte de la población. En Inglaterra, de 1958 a 1974, aproximadamente $75 \%$ de los niños se encontraban vacunados contra la tosferina. Sin embargo, para 1978 el porcentaje había declinado hasta $30 \%{ }^{37}$. Además, un editorial de "The Lancet ${ }^{30 "}$ " concluyó que para 1983 la vacuna antisarampionosa apenas era aplicada a poco más de $50 \%$ de la población infantil susceptible. El mismo edito- rial mostraba también que la causa principal de la baja cobertura era la falta de interés por el problema entre los médicos de las famílias quienes no promovían adecuadamente entre sus pacientes los beneficios de la vacuna. Por su parte, la baja cobertura de la vacuna antitosferínica fue atribuida a la difusión masiva de la información respecto al número de casos con complicaciones del sistema nervioso asociadas con ella ${ }^{10}$. Bate ${ }^{5}$ llevó a cabo un estudio entre 64 famílias para conocer su actitud ante la vacuna antitosferínica. Encontró que $52 \%$ de los padres sentían ansiedad respecto a ella y $25 \%$ de ellos fueran aconsejados por personal médico de no tomarla. Resultados semejantes obtuvo Adjaye ${ }^{1}$ mostrando que la causa más común para no aceptar la vacuna era el "consejo contra la vacuna" por parte de un profesional de la salud además de la presencia de alguna enfermedad en los niños antes de la inmunización y otros motivos referidos a olvidos, confusiones y factores relacionados.

Hull $^{24}$, al reflexionar sobre los resultados mostrados por otros autores, señaló que "la mayoría de los padres tienen una buena apreciación de los beneficios y los riesgos de la vacuna antitosferínica $\mathrm{y}$ aceptan que muchas cosas no se saben, pero quedan perplejos ante los mensajes confusos y contradictorios que obtuvieron de los consejeros locales de salud y de las autoridades nacionales". El citado autor sugiere que los programas de vacunación deberían ser apoyados por sistemas de comunicatión y educación para evitar caer en contradicciones sobre los mensajes enviados.

También en Inglaterra, Guest ${ }^{21}$ entrevistó a 112 padres de família y encontró que $17 \%$ tuvieron objeciones relacionadas con el miedo a los efectos colaterales de la vacuna lo que los llevó a no aceptar la DPT pero si a aceptar otras vacunas. Además del total de padres que no inmunizaban a sus hijos con DPT, $17 \%$ tonaron la decisión de manera personal y $14 \%$ lo hicieron después de haber recibido un consejo profesional.

\section{Modelos Psicologistas}

Uno de los aspectos que más se ha destacado en los países desarrollados está relacionado con el miedo a la vacunación y otras actitudes similares. Merrill ${ }^{31}$ estudió las actitudes de algunos grupos de población en el estado de California que había sido uno de los más afectados por el accident Cutter. A través de una encuesta encontró que las razones más comunes para no vacunar a los niños fueron: miedo e inseguridad ante la vacuna, la oposición general a cualquier vacuna, la oposición del padre (jefe de família) o del 
médico asesor a la vacuna y otras como la falta de motivación, el alto costo de la vacuna, y alguna enfermedad del niño al momento de recibir la vacuna.

Rosenstock ${ }^{39}$ mostró la importancia de algunos factores psicológicos. El primero de ellos se refería a las 'creencias' que tenían los individuos de ser susceptibles o no a la enfermedad bajo el principio de que "se sabe que el comportamiento es determinado más por la creencia que uno tiene de la realidad que por la realidad misma”. Más allá de la esfera individual, se mostraron elementos de la vida social que eran importantes, entre los que se contaban la presiones sociales y los bajos niveles de educación de algunos grupos.

A mediados de los 80 , utilizando elementos conceptuales de la psicología social, Buchner y col. ${ }^{9}$ y Montano ${ }^{32}$ sugirieron la posibilidad de construir modelos de actitudes para entender y predecir el comportamiento ante las vacunas. Tales modelos consideraban la incorporación de una gran cantidad de variables que podían determinar el comportamiento del individuo ante la oferta de la vacuna contra la influenza.

\section{ESTUDIOS EN PAÍSES EN VIA DE DESARROLLO}

A diferencia de los países industrializados, en los países en desarrollo se han encontrado dificultades específicas para lograr la cobertura universal. En este caso, la falta de participación se ha asociado a la existencia de síndromes culturales en las poblaciones entre los cuales destaca el miedo a la vacunación y la falta de información.

\section{Política Inmunizatoria}

En Singapur se encontró que a pesar de los esfuerzos por difundir el programa de vacunación antisarampionosa, la respuesta de la población había sido muy débil debido a la preferencia popular de permitir el desarrollo natural del sarampión en los niños. Ante la baja respuesta, las autoridades sanitarias iniciaron un programa de educación para la salud con el fin de "promover la inmunización y vencer (subrayado nuestro) las creencias de la cultura tradicional que ven al sarampión como una efermedad inocua ${ }^{16 "}$ ".

Olugbile ${ }^{36}$ encontró una baja cobertura vacunal especialmente para las vacunas BCG y antipolio. El trabajo muestra la relación entre el nivel de educación de los padres y el estado vacunal de los niños; a mayor educación mejor el estado vacunal. Sin embargo el autor señala que las diferencias entre grupos no se pueden explicar totalmente por el nivel educativo, ya que también otro factor a considerar era la accesibilidad a los servicios.

Madkour y Khalifa ${ }^{27}$ observaron en Egipto que a pesar de que en 1974 la vacuna BCG se había vuelto obligatoria en todas las gobernaturas egipcias, su cobertura era aún muy baja. En un estudio realizado en 1.138 niños de ubicación urbana, 55\% de ellos no habían recibido nunca la vacuna. Según los autores el bajo nivel de cobertura se debía a que no sólo las madres, sino también el personal médico eran contrarios a la vacuna BCG debido a lo frecuente de sus complicaciones.

Para México existen estudios específicos sobre el desarrollo de los programas de vacunación en poblaciones indigenas. De la Fuente ${ }^{12}$ señaló en 1953 que en la región Tzeltal-Tzotzil en los altos de Chiapas había resistencia para vacunar a los habitantes indígenas de la región para lo cual fue crucial la participación de los "enfermeros vacunadores chamulas, zinacantecos, huistecos y oxchuqueros, una de cuyas misiones básicas (era) ayudar a los médicos explicando y convenciendo".

Por su parte Aguirre-Beltrán² analizó el problema basado en experiencias de distintos grupos indígenas. Atribuyó también la falta de la aceptación de los conceptos y prácticas de la medicina científica a la existencia de conceptos y prácticas de la medicina indígena tradicional. No obstante, en particular para el caso de la aceptación de la medicina preventiva científica destacó el papel jugado por la actitud con la que los médicos y enfermeras trataban a sus pacientes indígenas; "su desdén por las creencias de la gente y su conducta agresiva e intransigente frente a las ideas y patrones de acción no racionales (sic) produjeron una barrera de resistencia a la introducción de la medicina moderna que fue de enorme trascendencia para el futuro desarrollo de las campañas preventivas".

\section{Culturas, Mitos y Miedo a Vacunar}

En Filipinas, Friede y col. ${ }^{18}$ utilizando métodos cuantitativos sofisticados trataron de determinar el 'riesgo' de los niños a no haber sido vacunados, basados en un modelo de comportamiento que considera factores demográficos, conceptuales o ideológicos y aquellos que facilitan o impiden el uso de los servicios. Del total de niños estudiados, $63 \%$ no estaban vacunados. Sin embargo, las razones más importantes de no vacunación que se encontraron fueron la negativa de los padres a vacunar a sus hijos, el miedo al dolor y a los efectos colaterales, la enfermedad del niño, el olvido, y la prohibición paterna. 
En este punto es importante observar el peso que los estudios atribuyen a la cultura y al folklore. Así, un buen ejemplo de ello es el siguiente. En 1964 en la región de Poona en la India se inició el programa de vacunación antipoliomielítica, lo cual fue aprovechado por Sathe y Shah ${ }^{40}$ para evaluar la respuesta de la población hacia ella. A través de la aplicación de un cuestionario estandarizado, se encontró que los factores que facilitaron la participación fueron los siguientes: miedo a la enfermedad y advertencia de la importancia de la vacunación $(73,7 \%)$, fé en la persona que sugirió la vacunación $(18,9 \%)$ y existencia de la vacuna gratuita $(7,4 \%)$.

A principios de la década de los 70, en la India el porcentaje de niños vacunados era muy bajo en opinión de algunos expertos ${ }^{4}$. Bhargava ${ }^{7}$ evaluó los logros de las campañas de vacunación masiva para esos años y encontró que la falta de cobertura se debía principalmente a deficiencias de su organización. De manera marginal comenta que las objeciones de la población y de los padres habían sido muy pocas, situación que sugería una amplia aceptación a los procedimientos vacunatorios. Atribuye los rechazos a las vacunas como signos de "ignorancia y miedo a recibir la vacunación”. Los resultados presentados por Mather y John ${ }^{29}$, un año más tarde, sobre un estudio realizado en la zona de Tamil Nadu, entran en franca contradicción con la opinión vertida por Barghava. Para estos autores, en muchas regiones de la India los conceptos sobre los factores que causan las enfermedades involucran fuerzas sobrenaturales; por lo que la modificación de estos factores puede causar la liberación de la ira de Dios con fatales consecuencias. En el mismo sentido, en Malasia, Chen ${ }^{11}$ reporta que entre la población de las zonas rurales en ese país, se cree que el tétanos neonatal es causado por un espíritu maligno. Por otra parte se sabe que durante la atención del parto, las comadronas ponen en práctica una serie de actos rituales con el fin de prevenir la llegada del espíritu, lo cual se considera asociado a la baja aceptación que manifiestan las madres de la vacuna antitetánica.

Una investigación más en Nigeria, también enfocada al estudio de la cultura médica popular ${ }^{35}$ en un grupo de 200 madres de família (90\% de ellas de origen Yoruba) muestra que $41 \%$ de las madres creían que la vacuna no servía porque conocían muchos casos de niños que aún habiendo sido vacunados habían contraido la enfermedad. El estudio concluye que el concepto Yoruba sobre el sarampión se encuentra dentro de una dimensión sobrenatural, bajo el argumento que en algunas de las víctimas quedaban con manchas después de un ataque severo, lo cual significaba que "el dios los había marcado".
En Bolivia, Bastien ${ }^{3}$ se interesó por determinar las percepciones culturales de la población respecto al tétanos y la vacuna antitetánica. Se realizó un estudio etnográfico en tres regiones basado en la observación participante y entrevistas estructuradas. Los resultados muestran que la población indígena en las tres regiones poseen teorías de la enfermedad con lo cual explican las causas del tétanos y las formas de terapia adecuadas. Bajo tales preceptos el tétanos no se define como una enfermedad específica sino como un síndrome que debe ser tratado fundamentalmente a través de la terapéutica tradicional indígena.

\section{Información Sobre las Vacunas}

Quizá este punto sea uno de los aspectos más importantes para entender la falta de participación en este grupo de países. En Nigeria se han realizado varios estudios sobre el tema. Este es el único país en desarrollo que se sabe planeó este tipo de estudios, previo al lanzamiento de los programas de 'erradicación' de la viruela y del control del sarampión. Las autoridades sanitarias se interesaron por saber el nivel de aceptación que se esperaría de la población. Los resultados del estudio piloto ${ }^{33}$ reconocieron algunos aspectos culturales como elementos importantes a estudiar. Las conclusiones del estudio resaltan la existencia de una gran confusión en la población sobre las causas y formas de prevención de la viruela y el sarampión. Cerca de $70 \%$ de las personas culparon a las condiciones climáticas de producir la viruela, otro porcentaje importante atribuyó el mal a la brujería y a la influencia del dios Shoponna. Respecto a la vacunación, $42 \%$ de los informantes señalaron que una de las razones por las que la gente no se vacunaba era porque no creían que ésta realmente protegiera contra la viruela. Sin embargo, relativamente pocos individuos mostraron oposición personal al programa de vacunación. En Ghana, Belcher y col. ${ }^{6}$ identificaron que la cobertura de la población era muy baja para casi todas las vacunas. Utilizando un cuestionario tipo CAP (conocimientos, actitudes y prácticas), previamente probado en otras poblaciones, obtuvieron información de 676 hogares. Las madres de los niños que no tenían vacunas (23\%) señalaron argumentos tales como que no contaban con información sobre las vacunas, que se habían olvidado de vacunar, que habían llegado tarde, que no hubo nadie que llevara al niño, que sintieron miedo, que creían que la vacuna no era efectiva, además de enfermedad del niño y de su corta edad para ser vacunado. 
Brown y col. ${ }^{8}$, en Camerún, utilizando un acercamiento etnográfico, estudiaron las causas por las cuales no se alcanzaban los niveles de participación poblacional esperados. En Yaounde, la capital del país, la baja cobertura se encontraba asociada a cuatro elementos: a ciertos vecindarios, a un grupo étnico en particular, a los bajos niveles socioeconómicos y las famíllias recién llegadas a la ciudad. Se utilizó una guía de preguntas abiertas para conocer opiniones sobre la vacunación en un grupo de famílias. Se encontró una gran falta de información sobre las enfermedades y las vacunas, derivado de una confusión considerable sobre los nombres de la enfermedad y sus síntomas debido al uso paralelo de dos lenguas nativas en la población y del francés por parte del personal de salud.

Factores semejantes fueron identificados por Lin y col. ${ }^{26}$ en un estudio en cinco comunidades en El Salvador. Los autores argumentan sobre la necesidad de hacer más accesible la información sobre los programas a la población con el fin de motivar su mayor participación.

En Bolivia, a principios de los 80, se evaluó el impacto del Programa Ampliado de Inmunizaciones $^{15}$. En el aspecto de participación de la comunidad en el programa se encontró que cerca de $70 \%$ de los niños menores de un año a quienes les fue administrada la primera dosis de vacuna DPT, no recibieron la tercera. Se encontró una actitud de resistencia a la vacunación y una alta tasa de abandono al programa en aquellas comunidades con una alta influencia de la cultura tradicional y/o e "bajo nivel sociocultural".

En 1983, en Honduras se llevó a cabo un estudio que incluyó a 275 madres de família en zonas rurales con el fin de identificar, a través de entrevistas estructuradas, los conocimientos, actitudes y prácticas referentes a inmunización ${ }^{42}$. Los resultados mostraron una falta de entendimiento por parte de las madres sobre los conceptos y las prácticas de inmunización, algunas de ellas no siendo capaces de entender el carácter preventivo de las vacunas, la edad de vacunación de los niños, además sintiendo desconfianza por sus efectos colaterales. El uso de las cartillas de vacunación para muchas madres con educación formal, resultó ser complicado mientras que para aquellas sin educación el manejo resultó totalmente incomprensible. Zelaya y $\mathrm{col}^{42}{ }^{42}$ sugieren que este tipo de problemas en la organización de las campañas de vacunación tienen que ser resueltos a través del diseño de programas de educación para la salud que consideren las características sociales y culturales específicas de las comunidades con las que se trabaja.
León ${ }^{25}$ también refiere conductas de resistencia de la población indígena ante la vacunas en Ecuador. Sin embargo, señala que a pesar de esta resistencia las campañas de vacunación contra la viruela, el sarampión, la poliomielitis, el tétanos y la tosferina, podrían considerarse exitosas y habían provocado una importante declinación de dichas enfermedades. A diferencia de éstas, la campaña contra la tuberculosis no había obtenido los mismos resultados debido en gran parte "al hacinamiento, la desnutrición y la ignorancia".

\section{CONCLUSIONES}

Los trabajos presentados invitan a una discusión sobre la posición metodológica de los investigadores, las técnicas empleadas y los factores identificados como motivo de no vacunación según grupo de países. En los países industrializados los métodos de elección son generalmente de tipo encuesta de opinión utilizando cuestionarios con preguntas cerradas. El acercamiento etnográfico ha sido poco utilizado a pesar de la experiencia de D'Onofrio ${ }^{14}$ en los años 60. La elección de una metodología con enfoque cuantitativo presupone que los investigadores conocen profundamente los elementos culturales que caracterizan a las poblaciones, lo cual les permite reducir la variabilidad de las posibles respuestas a un listado corto de opciones mutuamente excluyentes. La posición es cuestionable en el sentido de que dichos valores pueden variar en una población dada a partir de criterios como clase social, filiación étnica y otros ${ }^{23}$. En los países en desarrollo en cambio los métodos de elección son comunmente de corte etnográfico. El tipo de descripciones que ofrecen son distintos a los primeros y en ellos los elementos folklóricos de las culturas juegan un papel importante para explicar el comportamiento de la población. No obstante, en ambos casos los problemas asociados a la baja cobertura de los programas de vacunación se centran en la población minimizando el papel de las unidades de atención y del personal de salud.

Un aspecto relacionado a lo anterior es el papel que se le otorga a las "decisiones informadas" que toman las poblaciones para participar o no en un programa de vacunación. En países desarrollados el acceso a la información que las poblaciones tienen en general facilita su participación, pero por momentos se ha convertido en un obstáculo, ya que se llega al punto de cuestionar el valor preventivo de las vacunas. En ello han jugado un papel importante el 
personal de salud, particularmente los médicos. En países en desarrollo las poblaciones no tienen un acceso tan fácil a la información además de que su racionalidad generalmente no se encuentra tan familiarizada con las premisas del modelo biomédico. Esta combinación ha llevado a algunos investigadores a concluir que las costumbres, las ideas y la "ignorancia" de las poblaciones operan como barreras para la participación.

No es posible cuestionar los avances de los programas de vacunación en términos de cobertura. Sin embargo, se sabe que el éxito de estos programas hasta el momento ha dependido del montaje de programas verticalizados con gran apoyo de los medios de comunicación masiva ${ }^{34}$. Los mensajes canalizados a través de estos medios varían en su contenido pero no es posible asegurar que se conviertan en va-

\section{REFERENCIAS BIBLIOGRÁFICAS}

1. ADJAYE, N. Measles immunization: some factors affecting non acceptance of vaccine. Public Health (London), 95:185-8, 1981 .

2. AGUIRRE BELTRÁN, G. Programas de salud en la situación intercultural. México. Instituto Indigenista Interamericano, 1955.

3. BASTIEN, J. Percepciones culturales del tétanos neonatal y sugerencias para la programación. Bolívia Reach, 1988.

4. BASU, N. India's immunization programme. World Health Forum, 6, 1985

5. BATE, T. W.; DAVIES, A. G.; KEANE, S. M.; PEARCE, K. M. Failure to vaccinate. Lancet, 1:342, 1984.

6. BELCHER, D. W.; NICHOLAS, D. D.; OFOSU-AMAAH, S.; WURAPA, F. K. A mass immunization campaign in rural Ghana. Factors affecting participation. Public Health Rep., 93:170-6, 1978 .

7. BHARGAVA, S. Evaluation of methods for mass immunizations in children. Indian Pediatr, 9:378-83, 1972.

8. BROWN, J.; DIOGDOM, P.; MURPHY, K.; KESSENG, G.; HEYMANN, D. Identifying the reasons for low immunization coverage a case study of Yaounde (United Republic of de Cameroon). Rev. Epidém. Santé Publ., 30:35-47, 1982.

9. BUCHNER, D. M.; CARTER, W. B.; INVI, T. S. The relationship of attitude changes to adherence with influenza immunuzation: a prospective study. Med Care, 23:771-9, 1985.

10. BURNEY, P. G. \& COOK, D. G. Mother's intentions and the immunization of their infants. J. R. Coll. General Pract., 229-32, 1983

11. CHEN, P. C. Y. The traditional birth attendant and neonatal tetanus: the Malaysian experience. J. Trop. Ped., 22:263-4, 1976. lores arraigados de la cultura de las poblaciones de cada país. De hecho, su corta temporalidad ha sido demostrada en distintas ocasiones tanto en países industrializados como en vías de desarrollo. Vale la pena entonces reflexionar e insistir sobre la responsabilidad que tiene el sector salud de generar una cultura preventiva, informada, sobre los beneficios y riesgos potenciales de las vacunas. Sin duda en la actualidad los usuarios de servicios de salud tanto personales como preventivos demandan cada vez más información sobre las opciones disponibles. Los programas de vacunación en el futuro deberán considerar que informar apropiadamente a la población sobre las vacunas es una buena forma de garantizar su participación.

En el cuadro Anexo se resumen los motivos principales que llevan a la población a no vacunar a sus niños.

12. DE LA FUENTE, J. El centro coordinador tzeltal-tzotzil. América Indígena, 13:55-64, 1953.

13. DIANESE, G. Treatment of whooping-cough. Lancet, 2:1224, 1982.

14. D'ONOFRIO, C. Reaching our hard-to-reach: the unvaccinated. 1965. [Mimeografado].

15. EXPANDED PROGRAMME ON IMMUNAZATION: evaluation of immunization coverage. Weekly. Epid. R., 57 (10): 85-6, 1982.

16. EXPANDED PROGRAMME ON IMMUNAZATION: programme acceleration.Weekly. Epid. R., 61 (48): 372-3, 1986.

17. FREEMAN, P.; JHONSON, K.; BABCOCK, J. A health challenge for the states: Achieving full benefit of childhood immunization. Boston, The John Mc Cormack Institute of Public Affairs, University of Massachusets, 1993.

18. FRIEDE, A.; WATERNAUX, C.; GUYER, B. An epidemiological assessment of imunization programme participation in the Phillipines. Int. J. Epidemiol., 14:135-41, 1985.

19. GLASSER, M. A. A study of the public's aceptance of the Salk Vaccine Program.Am. J. Public Health, 48:141-6, 1958.

20. GRANDOLFO, M.; SANTORO, R.; POLO, M.; SCARDELLATO, V.; ALBERT, A.; PASQUINI, P. A pilot measles vaccination campaign in Italy. Public Health, 100:208-13, 1986.

21. GUEST, M. \& HORN, J. Why some parents refuse pertussis immunization. Practitioner, 230:210, 1986.

22. HEGGENHOUGEN, H. K. \& CLEMENTS, J. Acceptability of childhood immunization. London, LSHTM, 1987. (EPC. Publication no 14).

23. HELMAN, C. Culture, health and illness. London, Butterworth - Heinemann, 1994. 
24. HULL, A. \& NICOLL, A. Immunization misinformation. Lancet, 2:1215-6, 1984.

25. LEÓN, L. Salud y medicina en los grupos aborígenes del oriente ecuatoriano. América Indígena, 37:(2), 1977.

26. LIN, N.; HINGSON, R.; ALLWOOD-PAREDES, J. Mass immunization campaign in El Salvador, 1969. Health Rep., 86:1113-21, 1971.

27. MADKOUR, M. \& KHALIFA, S. A critical review of BCG vaccination programme in Egypt: final report, 1977.

28. MARCUSE, E. Immunization: an embarrasing failure. Pediatrics, 56:493-4, 1975.

29. MATHER, R. J. \& JOHN, T. J. Popular beliefs about smallpox and other common infectious diseases in south India. Trop. Geogr. Med., 25:190-6, 1973.

30. MEASLES and primary health care. [Editorial]. Lancet, 1:1275-6,1984.

31. MERRILL, M. H.; HOLLISTER, A. C.; GIBBENS, S. F.; HAYNES, A. W. II. Attitudes of californians towards poliomyelitis vaccination. Am. J. Public Health, 48:146-52, 1958.

32. MONTANO, D. Predicting and understanding influenza vaccination behaviour. Med. Care, 24:438-53, 1986.

33. MORGAN, R. Attitudes towards smallpox and measles in Nigeria. Int. J. Health Educ., 12:77-85, 1969.
34. NICHTER, M. Vaccination in South Asia: false expectations and commanding metaphors. In: Coreil, J. \& Mull, D. ed. Anthropology and primary health care. Boulder Westriew Press, 1990.

35. ODEBIYI, A. I. \& EKONG, S. C. Mothers' concept of measles and attitudes towards the measles vaccine in Ile-Ife, Nigeria. J. Epidemiol. Community Health, 36:209-13, 1982.

36. OLUGBILE, A. A study of the preventive health services in Ibadan with special reference to the immunization of preschool children. Nigeria Med. J., 5:(2), 1974.

37. POLLARD, R. Relation between vaccination and notification rates for whooping cough in England and Wales. Lancet, May 31, 1980.

38. PROFETA, M. L.; FERRANTE, P.; PORRO DE'SOMENZI, C. A survey on factors affecting acceptance of measles vaccine. Eur J. Epidemiol., 2:128-33, 1986.

39. ROSENSTOCK, I.; DERRYBERRY, M.; CARRIGER, B. Why people fail to seek poliomyelitis vaccination. Public Health Rep., 74:98-103, 1959.

40. SATHE, P. V. \& SHAH, V. Parental participation in polio vaccination programme. Indian J. Public Health, 9:, 1965.

41. WISHIK, S. Attitudes and reactions of the public to health programs. Am. J. Public Health, 48:139-41, 1958.

42. ZELAYA, J.; MATA, J.; MILLS, E. Bridging the communication gap. Assign. Child., 69/72:, 1985. 